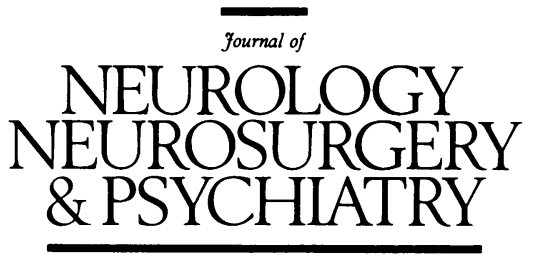

Editorial

\title{
Progress in the treatment of epilepsy
}

It has been generally supposed, both by the medical profession and by the public, that the prognosis of epilepsy is poor. This arises both from the impression created by multiple handicapped persons with epilepsy, and from formal research studies in patients whose chronicity had brought them to specialised epilepsy centres. ${ }^{1}$ In fact, prospective and community-based studies over the past decade show that as many as $\mathbf{8 0} \%$ of newly diagnosed patients achieve prolonged remission, most being seizurefree within two years of starting treatment. ${ }^{23}$ It also emerges from these studies that a major determinant of prognosis is the number of seizures, or the time elapsing before the start of therapy. ${ }^{45}$ The aphorism of Gowers ${ }^{6}$ that seizures beget seizures appears to be of crucial importance in the early stages of treating epilepsy. If epilepsy is a liability to recurring seizures, it cannot be diagnosed after a single attack. However, if one apparently unprovoked attack carries a high risk of recurrence, and if early treatment prevents chronicity, it may be unreasonable to withhold medication until there is recurrence. There is no consensus over the probability of epilepsy after a single seizure: estimates range from $27 \%$ to $71 \%,{ }^{78}$ probably reflecting different patterns of referral: as it is common for any second attack to rapidly follow the first, the prognosis of single seizures appears better if assessment is delayed. ${ }^{910}$

As chronicity is not the usual outcome of epilepsy, therapeutic failure should not be accepted as inevitable without thorough reassessment of both diagnosis and treatment. Among patients admitted for assessment of resistant epilepsy, more than $10 \%$ prove to be suffering from pseudoseizures, ${ }^{11}$ alone, or in combination with well controlled epilepsy. Where this possibility arises intensive monitoring with video recording of clinical events and EEG telemetry may be required to establish the diagnosis. Monitoring is also an important factor in the overall reassessment of intractable seizures, often leading to reclassification of the syndrome and selection of more appropriate medication. ${ }^{1211}$

The antiepileptic drugs (AEDs) which prove effective in most patients with epilepsy are less than satisfactory because of side effects. Some degree of sedation is usual with current treatment and, particularly in schoolchildren, may represent a source of psychosocial dysfunction no less important than the seizures. For many years it was the received wisdom in the pharmaceutical industry that AEDs were orphan drugs, but the past decade has seen intense activity in the development of new agents. In part this has been encouraged by advances in neurochemistry. Most attention has focused on drugs facilitating GABA-ergic inhibitory neurotransmission, although currently calcium entry blockers and inhibitors of excitatory transmission are being evaluated. Clinical testing of AEDs is difficult, and concern with trial design is only a recent development. Reviews in 1968,1976 and 1982 identified respectively 2,6, and 24 methodologically acceptable controlled AED trials. ${ }^{13-15}$ Because there is generally no justification for withholding or delaying treatment of epilepsy, early placebo-controlled trials of monotherapy would be unethical. Phase II testing of new AEDs is based on add-on therapy in resistant patients with a high seizure frequency. This approach conflicts with the current view that monotherapy should be used wherever possible, and that adding a second drug rarely improves seizure control. ${ }^{16}$ Thus new drugs are evaluated under highly unfavourable conditions and there is every possibility of a new agent, as effective as phenytoin but less toxic, being rejected as inactive. Moreover, pharmacokinetic interactions may alter metabolism of either the test drug or co-medication, hindering accurate adjustment of plasma levels, and possibly confusing assessment of both efficacy and tolerability. The International League against Epilepsy took an early initiative in proposing guidelines for AED trials, ${ }^{18}$ and no new solutions have since been found to the methodological problems.

Three promising new drugs have recently completed phase II testing. Vigabatrin, an inhibitor of GABA transaminase, has shown high efficacy in add-on trials of patients with therapy resistant epilepsy, more than $50 \%$ of whom have shown a seizure reduction of more than $50 \%{ }^{19}$ Because the drug attaches itself irreversibly to the enzyme, plasma levels are non-critical and dosing correspondingly simple. Some concern has been caused by the occurrence of reversible microvacuolation in the white matter of rats, mice and dogs, but this has not been established in primates nor has vacuolation been found in humans, either in surgically resected material or at necropsy examination. Lamotrigine, acting most probably by inhibition of glutamate release, is the first AED drug which acts by antagonism of excitatory neurotransmission to complete extensive clinical testing. Side-effects are few and largely 
avoidable at therapeutic plasma concentrations, moderate but significant efficacy has been demonstrated in add-on trials, chiefly in patients with therapy resistant partial epilepsies. ${ }^{2021}$ The kinetics are linear and bioavailability $100 \%$, and the drug does not cause enzyme induction; its metabolism is, however, influenced by co-medication which must be taken into account in dosing. A third new AED at an advanced stage of clinical evaluation is oxcarbazepine, closely related to carbamazepine but without the potential of the latter to cause hepatic enzyme induction, accelerating both its own metabolism and that of comedication. Its efficacy appears similar to that of carbamazepine for the treatment of partial and generalised tonic-clonic seizures, but severe side-effects are less common.

As long as epilepsy is sometimes refractory to medication, there will be a significant place for surgery. As a recent leader in the Lancet $^{22}$ pointed out, surgical treatment is indicated in a larger proportion of patients than is generally realised. The possibilities for identifying and successfully treating epileptogenic foci have been increased by recent developments in intracranial recording and by new surgical techniques. Selective amygdalo-hippocampectomy, ${ }^{23}$ avoiding removal of neocortex, produces no obvious cognitive deficit, and indeed may be successful in patients where conventional temporal lobectomy is contraindicated on neuropsychological grounds. Multiple subpial transection $^{24}$ involves the division of epileptogenic cortex into thin vertical slices. Establishment and propagation of epileptic discharges depends largely on tangential fibres whereas the most important functional connections of the cortex are radial. Thus it is possible to control partial seizures arising in regions such as the motor cortex and Broca's area, which could not be excised without producing unacceptable deficits.

Of the alternatives, producing more effective, less toxic antiepileptic drugs, advancing epilepsy surgery, or preventing the development of chronic epilepsy, clearly the last is the most attractive option. There is therefore an urgent need for further research concerning the natural history of epilepsy, the determinants of prognosis, and the consequences of early treatment.

The Maudsley Hospital, Denmark Hill, London

C D BINNIE

1 Rodin EA. The prognosis of patients with epilepsy. Springfield: Thomas, 1968. 2 Annegers JF, Hauser WA, Elveback LR. Remission of seizures and relapse in patients with epilepsy. Epilepsia 1979;20:729-37.

3 Elwes RDC, Johnson AL, Shorvon SD, Reynolds EH. The prognosis for seizure control in newly diagnosed epilepsy. New Engl J Med 1984;311:944-7.

4 Shorvon SD, Reynolds EH. Early prognosis of epilepsy. Br Med J 1982;2:1023-5.

5 Reynolds EH. Early treatment and prognosis of epilepsy. Epilepsia 1987;28:47-106

6 Gowers WR. Epilepsy and other chronic convulsive diseases: their causes, symptoms and treatment. London: William Wood, 1881:309.

7 Hauser WA, Anderson VE, Loewenson RB, McRoberts SM. Seizure recurrence after a first unprovoked seizure. New Engl J Med 1982;307:522-8.

8 Elwes RDC, Chesterman P, Reynolds EH. Prognosis after a first untreated tonic-clonic seizure. Lancet 1985;2:752-3.

9 Hopkins A, Gorman A, Clark C. The first seizure in adult life: the value of clinical features, electroencephalography and computerised tomographic scanning in predicting seizure recurrence. Lancet 1988;1:721-6.

10 Reynolds EH. A single seizure. Br Med J 1988;297:1422-33.

11 Binnie CD. Ambulatory diagnostic monitoring of seizures in the adult. In: Gumnit RJ ed. Advances in Neurology 46: International Conference on Neurodiagnostic Monitoring. New York: Raven Press, 1987:169-82.

12 Sutula TP, Sackellares JC, Miller JQ, Dreifuss FE. Intensive monitoring in refractory epilepsy. Neurology (NY) 1981;31:243-7.

13 Coatsworth JJ. Studies of the clinical efficacy of marketed antiepileptic drugs. 1971; Washington: US Government Printing Office 1971:36.

14 Richens A. Drug treatment of epilepsy. London: Henry Kimpton, 1976:176.

15 Gram L, Drachmann Bentsen K, Parnas J, Flachs H. Controlled trials in epilepsy: a review. Epilepsia 1982;23:491-519.

16 Hakkarainen H. In: Akimoto H, Kazamatsuri H, Seino M, Ward AA, eds. Advances in Epileptology: XIIIth Epilepsy International Symposium. New York: Raven Press, 1982.

17 Schmidt D. Two antiepileptic drugs for intractable epilepsy with complexpartial seizures. J Neurol Neurosurg Psychiatry 1982;45:119-24.

18 Penry JK. Principles for clinical testing of antiepileptic drugs. Epilepsia 1973;14:451-8.

19 Treiman DM. Gamma vinyl GABA: current role in the management of drug-resistant epilepsy. Epilepsia 1989;30(Suppl 3):S31-5

20 Binnie CD, Debets RMC, Engelsman M, et al. Double-blind crossover trial of Lamotrigine (Lamictal) as add-on therapy in intractable epilepsy. Epilepsy Res 1989;4:222-9.

21 Jawad S, Richens A, Goodwin G, Yuen WC. Controlled trial of Lamotrigine (Lamictal) for refractory partial seizures. Epilepsia 1989;30:356-63

22 Leading Article. Surgery for temporal lobe epilepsy. Lancet 1988;ii:1115.

23 Yasargil MG, Teddy PG, Roth P. Selective amygdalohippocampectomy. Operative anatomy and surgical technique. In: Syman L, eds. Advances and technical standards in neurosurgery Vol 12. Wien: Springer-Verlag, 1985:93-123.

24 Morrell F, Whisler WW, Bleck T. Multiple subpial transection: a new approach to the surgical treatment of focal epilepsy. $J$ Neurosurg 1989;70:231-9. 